\title{
Erratum: Two-Dimensional Monomer-Dimer Systems are Computationally Intractable ${ }^{1}$
}

\author{
Mark Jerrum ${ }^{2}$
}

Received December 18, 1989

I am greatly indebted to Scott Provan for pointing out a flaw in one of the constructions employed in the above paper. The flaw lies in the graph $\Delta_{2}$ presented in Fig. 2. This graph is intended, in the terminology of the paper, to have matching polynomial $P\left(A_{2} ; x, y, z\right)=2(1+x y z)$. In fact, the matching polynomial of $\Delta_{2}$ also includes some spurious degree-2 terms, in contradiction of Eq. (3).

To correct the construction, the graph depicted in the original Fig. 2 should be augmented with three extra edges, so that it corresponds with the revised Fig. 2 given here. The matching polynomial of the revised

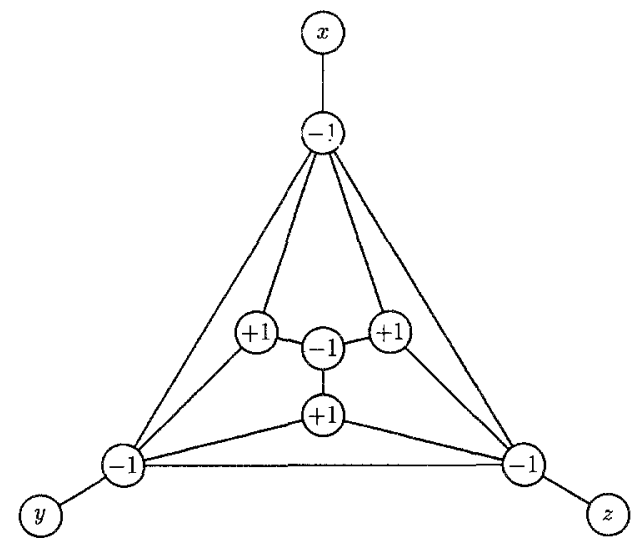

Fig. 2 (revised). The graph $\Delta_{2}$.

\footnotetext{
${ }^{1}$ This paper originally appeared in J. Stat. Phys. 48:121-134 (1987).

${ }^{2}$ Department of Computer Science, University of Edinburgh, Edinburgh EH9 3JZ, United Kingdom.
} 
version of $\Delta_{2}$ is now exactly as claimed in Eq. (3), and no changes are necessary in the text of the paper. However, the graph $\Gamma$ depicted in Fig. 3 contains three copies of the flawed version of $\Delta_{2}$, and hence should be modified accordingly. I apologize to any reader who was prevented, by this error, from following the detailed argument of the paper. 